\title{
Prenatal care disruptions and associations with maternal mental health during the COVID-19 Pandemic
}

1 Taylor Groulx ${ }^{1,5+}$, Mercedes Bagshawe ${ }^{1,2,5+}$, Gerald Giesbrecht PhD, R.Psych. ${ }^{1,3,4}$, Lianne

2 Tomfohr-Madsen, PhD, R.Psych. ${ }^{1,3,4}$, Erin Hetherington, PhD ${ }^{6}$, Catherine Lebel, PhD ${ }^{1,2,5^{*}}$

3 'Alberta Children's Hospital Research Institute, University of Calgary, Calgary, Alberta, Canada

$4 \quad{ }^{2}$ Hotchkiss Brain Institute, University of Calgary, Calgary, Alberta, Canada

$5 \quad{ }^{3}$ Department of Psychology, University of Calgary, Calgary, Alberta, Canada

$6 \quad{ }^{4}$ Department of Pediatrics, University of Calgary, Calgary, Alberta, Canada

7 5epartment of Radiology, University of Calgary, Calgary, Alberta, Canada

$8 \quad{ }^{6}$ Department of Obstetrics and Gynaecology, University of Calgary, Calgary, Alberta, Canada

$9 \quad{ }^{+}$These authors contributed equally to this work.

10

11 * Corresponding Author:

12 Dr. Catherine Lebel, PhD

13 Alberta Children's Hospital

1428 Oki Dr NW

15 Calgary, AB T3B 6A8

16 clebel@ucalgary.ca

17

18 Word Count: 2,252

19 \# of Figures: 0

20 \# of Tables: 2 
Abstract

As the novel coronavirus (COVID-19) spread across Canada in March 2020, provinces imposed strict lockdowns causing restrictions and disruptions to health care. These changes impacted how pregnant individuals received prenatal care and experienced childbirth. The additional stress caused by these changes may negatively affect the well-being of pregnant individuals and the developing child. This study investigated the impact of the pandemic on prenatal care and birth plans of pregnant individuals in Canada and potential associations with maternal mental health. Data from 4604 participants was collected from English- and French-speaking Canadians between April 5-June 1, 2020 as part of the Canada-wide Pregnancy During the COVID-19 Pandemic study. Symptoms of maternal depression, general anxiety, and pregnancy-related anxiety were assessed. Participants also answered questions about disruptions and changes to prenatal care and their birth plans due to the COVID-19 pandemic. Logistic regression was used to estimate associations between prenatal care disruptions and maternal mental health. Cancellation of prenatal appointments and birth plan changes (specifically changes to childcare during birth and change of support person attending the birth) were significantly associated with greater odds of experiencing clinically-elevated depression, anxiety, and/or pregnancy-related anxiety symptoms. These results illustrate the need for reliable and accessible prenatal care during the pandemic, such as the integration of mental health screenings and

38 co-ordination of prenatal care providers.

39 Keywords: pregnancy, prenatal care, pandemic, depression, anxiety, COVID-19, stress 
Proper prenatal care is important for the health of both the pregnant individual and the developing baby. Inadequate prenatal care has been associated with low birth weight, preterm birth, and miscarriage (1-5). The emergence of the novel coronavirus (COVID-19) led to widespread lockdowns that disrupted prenatal care for many pregnant individuals around the world. During the COVID-19 pandemic, the Canadian government recommended remote appointments with doctors and obstetricians for pregnant individuals where possible (6), and some hospitals and doctors' offices restricted support persons from prenatal appointments, ultrasounds or the birthing room, switched to virtual appointments and limited in-person meetings with the care team. Restrictions and changes to prenatal care and birth protocols occurred quickly, potentially adding to the uncertainty and stress for pregnant individuals. Given these regulations to restrict appointments and visits from family and friends, it is conceivable that pregnant individuals may perceive a diminished level of support, which is directly associated with increased depressive symptoms (7) and postnatal distress (8).

Prior studies suggest that $10-25 \%$ of individuals experience mild to moderate anxiety and/or depression during pregnancy (9). Prenatal depression and anxiety have been linked to a negative perception of the birth experience (10), greater risk of postnatal depression (11-14) and loss of interest in the child (15). Prenatal stress is also related to adverse cognitive and behavioural outcomes in children after birth (16-19). Pregnancy-related anxiety refers to worries or fears in relation to childbirth, the safety and the health of the baby, and future parenting. Pregnancy-related anxiety is strongly associated with adverse birth outcomes, including preterm birth and low birth weight, problematic infant temperament, behavioural and emotional problems in the child, and

62 developmental delays (20-22). Recent studies indicate substantially elevated psychological distress in pregnant individuals during the current COVID-19 pandemic compared to similar pre-pandemic pregnancy cohorts, as indicated by elevated depression and anxiety symptoms (23-27).

With the restrictions in place to combat COVID-19, and the elevated anxiety and depression symptoms in this population, the goal of the present study was to determine the impact of the COVID-19 pandemic on prenatal care and birth plans of pregnant individuals in Canada and how these changes may be associated with maternal mental health.

\section{Materials and Methods}

\subsection{Participants}

The current study reports data collected from the Pregnancy during the COVID-19 Pandemic study (28) between April 5 - June 1, 2020. Data between these dates was chosen for analysis to reflect the effects of the initial lockdown period. This ongoing study recruited pregnant individuals across Canada by use of social media to complete an online survey. The inclusion criteria were as follows: living in Canada, able to read and write English and/or French, 17 years of age or older, and having a confirmed pregnancy $<35$ weeks' gestation. This study was approved by the University of Calgary

77 Conjoint Health Research Ethics Board (REB20-0500).

\subsection{COVID-19 and Prenatal Care}

79 Participants completed a questionnaire about disruptions and changes to their prenatal care and birth plan due to the current COVID-19 pandemic. Participants were presented with the following questions/statements: "Have you experienced changes in the way that prenatal care is delivered to 
cancelled?", and "Are you able to bring your partner or support person to your appointments?" Participants answered these questions using a binomial scale (yes/no). Participants were also asked "Which changes have you made to your birth plan (check all that apply): Birth Location; Support People; Childcare Arrangements; Other changes".

\subsection{Anxiety and Depression Symptoms}

The Edinburgh Postnatal Depression Scale (EPDS) (29-30) was used to measure maternal depressive symptoms. Although it is not a diagnostic tool, scores of 13 or more are associated with a diagnosis of depressive disorder (29). The PROMIS Anxiety Adult 7-item short form was used to assess general anxiety. Scores of 60 or more on this measure have been associated with clinically elevated anxiety (31). Data from the EPDS and PROMIS measures were dichotomized at the established cutoff scores, with scores $\geq 13$ and $\geq 60$ respectively, representing clinically elevated symptoms. Pregnancy-related anxiety, referring to fear and worries surrounding the circumstances of birth and health of baby, was assessed differently in the English and French sample due to availability of measures. Responses to the English survey were assessed using a 10-item questionnaire (PRAQ), in which participants pick from four possible responses. Responses to the French survey were assessed using a similar, but slightly different validated 10-item questionnaire (PRAQ-R2), in which participants chose from five responses per question (33). Neither the PRAQ (English) nor the PRAQR2 (French) provides cut-off scores for clinically elevated symptoms, so the upper quartile of each was used as a cut off for these measures to indicate relevant elevations in pregnancy-related anxiety (PRAQ $\geq 24$ and PRAQ_R2 $\geq 30$ ).

\subsection{Statistical Analysis}

Survey responses were manually checked for accuracy before analysis and invalid records (e.g., implausible due date) were removed. The associations between clinically elevated psychological symptoms and COVID-19 related disruptions to prenatal care and birth plans were estimated using multivariable logistic regression using IBM SPSS. Separate logistic regressions were completed for each measure of mental health in relation to prenatal care disruptions and changes to birth plans. The PROMIS Anxiety and EPDS measures were analyzed from English and French survey responses in combination, as the same validated measure was used in both languages. Analysis for the PRAQ and PRAQ-R2 were completed separately for participants completing the survey in English or French, as both validated measures consist of slightly different questions and different score ranges. Logistic regression models were adjusted for age, parity, ethnicity, trimester, maternal education and household income as covariates. Missing data for any value was addressed as N/A and those values did not contribute to the statistical model. IBM SPSS Statistics 26 was used for all statistical analysis.

\section{$3 \quad$ Results}

\subsection{Participants}

A total of 4604 records collected between April 5 - June 1, 2020 were included in the current analysis. 3755 participants completed the survey in English and 849 in French. Participants were aged $31.56+/-4.39$ years (range 18-49 years). The majority of participants were married (67.0\%) or living with a partner (28.6\%). All participants lived in Canada, with the majority residing in Ontario (28.3\%), Alberta (23.2\%), and Quebec (21.0\%). Most participants self-identified as Caucasian $(81.6 \%)$, with others identifying as First Nations (1.0\%), Metis (1.2\%), Black (1.7\%), Chinese (1.7\%), Filipino (1.4\%), Korean (0.2\%), West Asian (0.6\%), South Asian (3.3\%), South-east Asian (0.3\%), Hispanic (2.1\%), and Mixed (4.9\%). 
The majority of participants reported their highest level of education as having completed a trade or community college diploma (24.0\%), a bachelor's degree $(40.3 \%)$, or a master's degree $(18.6 \%)$. Participants had a median income range of CAD $\$ 100,000-124,999$ per year (USD $\$ 75,000$ 95,000). 46.5\% (2143/4604) of all participants reported that this was their first pregnancy. $45.6 \%$ of participants reported having other children $(32.9 \%$ had one child, $9.6 \%$ had two children and $3.2 \%$ had three children or more). Average gestation of participants when they completed the survey was $21.63+/-8.48$ weeks (range 3.7-35).

\subsection{Prevalence of Mental Health Symptoms}

Mean scores on mental health measures can be found in Table 1. 34.0\% of participants experienced clinically-elevated symptoms of depression (EPDS score $\geq 13$ ), and $70.9 \%$ of participants had clinically-elevated symptoms of anxiety (PROMIS score $\geq 60$ ).

\section{$137 \quad 3.3$ Disruptions and Changes to Prenatal Care}

$13889 \%$ of participants reported changes in the way that prenatal care was delivered to them during the 139 COVID-19 pandemic (Table 1). 40\% of respondents reported the cancellation of at least one prenatal care appointment and $90.9 \%$ of participants were not permitted to bring their partner or support person to prenatal appointments. Regarding changes to the birth plan due to the COVID-19 pandemic, $33.4 \%$ of participants made changes to their birth plan: $9.4 \%$ reported making a change to their birth location, 26.7\% reported making a change to their birth support person(s), and 10.9\% reported making a change to their childcare plan (some participants made changes to multiple factors and thus numbers do not add to $33.4 \%$ ).

\subsection{Statistical Analysis}

Complete details of the statistical models may be found in Table 2. Cancellation of prenatal appointments was associated with increased odds of a participant experiencing clinically elevated depression symptoms, $\mathrm{OR}=1.36,95 \% \mathrm{CI}[1.19,1.56], \mathrm{p}<.001$, and clinically elevated general anxiety, OR $=1.33,95 \%$ CI $[1.17,1.52], \mathrm{p}<.001$. Changes to the planned support person(s) attending the birth increased odds of a participants score falling above the cut-off on depressive symptoms, OR $=1.61,95 \% \mathrm{CI}[1.36,1.91], \mathrm{p}<.001$, and general anxiety symptoms, OR $=1.77,95 \% \mathrm{CI}[1.49,2.09]$, $\mathrm{p}<.001$. Changes to planned childcare arrangements during labor increased the odds of participant scores falling above the cut-off on depressive symptoms, $\mathrm{OR}=1.50,95 \% \mathrm{CI}[1.18,1.92], \mathrm{p}=.001$, and anxiety symptoms, $\mathrm{OR}=1.48,95 \% \mathrm{CI}[1.16,1.90], \mathrm{p}=.002$. Among English responses, odds of pregnancy-related anxiety within the upper quartile were increased by cancelation of prenatal appointments, $\mathrm{OR}=1.39,95 \% \mathrm{CI}[1.17,1.64], \mathrm{p}<.001$; changes to planned support person(s), $\mathrm{OR}=1.70,95 \% \mathrm{CI}[1.39,2.08], \mathrm{p}<.001$; and changes to childcare during labor, $\mathrm{OR}=1.46,95 \% \mathrm{CI}$ $[1.08,2.00], \mathrm{p}=.015$. Of French responses, only changes to the planned support person(s) able to attend the birth was associated with significantly increased odds of pregnancy-related anxiety symptoms in the upper quartile, OR $=1.58,95 \%$ CI $[1.00,2.50], \mathrm{p}=.049$.

\section{Discussion}

163 Stress during pregnancy increases the risk of physical and psychological problems in both the 164 pregnant individual and the child (7, 34-35). The uncertainty stemming from the COVID-19 165 pandemic, and specifically changes related to the delivery of prenatal care may contribute 166 to higher stress in pregnant individuals, thereby increasing the risk of adverse 167 psychological outcomes such as depression or anxiety (36-38). The results of this study suggest that 
168

169

170

171

172

173

174

175

176

177

178

179

180

181

182

183

184

185

186

187

188

189

190

191

192

193

194

195

196

197

198

199

200

201

202

203

204

205

206

207

208

209

210 disruptions to prenatal care and birth plans stemming from COVID-19 are associated with clinically elevated symptoms of depression and anxiety in a large sample of pregnant individuals across Canada. These findings correspond with recent studies showing elevated depression and anxiety in pregnant individuals during the COVID-19 pandemic (23-27).

Eighty nine percent of pregnant individuals in this study reported at least one pandemic-related change in the delivery of prenatal care. Of these changes, the most prevalent were inability to have support persons attend prenatal appointments (90.6\% of participants) and cancellations of prenatal care appointments (40\% of participants). COVID-19 related changes in prenatal care may contribute to an atmosphere of uncertainty around maternal and fetal health, which may compound the already elevated levels of stress, further increasing the risk of psychological distress. Care providers should work to provide pregnant individuals with consistent and informed prenatal care in an effort to reduce uncertainty and stress.

This study demonstrates that cancellations of prenatal appointments, changes to support person(s) at the birth and changes to childcare arrangements during labor are significantly associated with psychological distress (i.e., clinically-elevated symptoms of depression and anxiety) of pregnant individuals during the pandemic. Given the benefits of support during pregnancy $(34,39)$, when considered in conjunction with the elevated levels of psychological distress in this population, the need for consistent and supportive care from medical professionals and the prenatal care team should be considered of utmost importance.

Cancellations of prenatal appointment(s), changes to the planned support person(s) attending the birth and changes to childcare plans during labor are all associated with elevated pregnancy-related anxiety in the subset of English respondents, while only a change in support person(s) attending the birth was associated with elevated pregnancy-related anxiety in the French subset. These distinctions between the English and French subsamples potentially reflect the differences in questionnaires used, the smaller sample size of French-speaking participants, or differences in how prenatal care is delivered or perceived in the province of Quebec compared to the rest of the country. Care should be taken to ensure an individualized approach to prenatal care during the COVID-19 pandemic to mitigate pregnancy-related anxiety in a manner which is specific to the individual in question.

Due to the COVID-19 pandemic, many forms of medical care have transitioned to telehealth (40). Remote delivery of prenatal care without the face-to-face experience has the potential to be perceived as less supportive, despite being an adequate substitute for complete cancellation $(40,41)$. When prenatal appointments were in person, most participants in this study were not allowed to bring their partner or support person, which may also contribute to a perceived decrease in support. In order to maintain high quality of care in the face of a public health crisis, prenatal and medical care professionals should ensure that they are delivering high-quality care in a personal way that maximizes support felt by the individual, which may require additional training in remote methods of care delivery.

Given that the questionnaires used in this study to quantify psychological distress are screening measures and not diagnostic tools, in-person clinical assessment may provide further insight into the psychological well-being of pregnant individuals. Furthermore, many participants noted that they were unsure what to expect for the remainder of their pregnancies. Future studies in similar populations should examine perceived changes to prenatal care while also considering changes associated with each stage of pregnancy. 
211 Pregnant individuals are experiencing significant psychological distress during the COVID-19

212 pandemic (23-27). Our research provides new insight into the relationship between COVID-19

213 disruptions to prenatal care and elevated maternal mental health problems. Our findings reveal high

214 rates of prenatal care disruptions faced by pregnant individuals during the pandemic. This study

215 illustrates the need for reliable and accessible prenatal care during the current pandemic, including

216 integration of mental health screenings and modified birth plans that create a supportive birthing

217 environment and are person-centered. Care providers should work together to provide pregnant

218 individuals with consistent prenatal care in order to reduce uncertainty.

\section{Acknowledgments}

220 We would like to thank all individuals who participated in the Pregnancy During the COVID-19

221 Pandemic study. This research was supported by the Alberta Children's Hospital Research Institute 222 and the Owerko Centre. 
223 Table 1: Demographics, Mental Health Scores, and Disruptions to Prenatal Care

\begin{tabular}{|c|c|c|c|c|}
\hline Measure & $\mathbf{n}$ & Mean & $\begin{array}{l}\text { Standard } \\
\text { deviation }\end{array}$ & Range \\
\hline Gestation (weeks) & 4604 & 21.63 & 8.48 & $3.7-35$ \\
\hline Age (years) & 4606 & 31.56 & 4.39 & $18-49$ \\
\hline \multicolumn{5}{|l|}{ Mental Health Scores } \\
\hline Edinburgh postnatal depression scale (EPDS) & 4491 & 10.30 & 5.37 & $0-30$ \\
\hline PROMIS anxiety T-Scores & 4477 & 58.74 & 8.2 & $36-82.7$ \\
\hline \multicolumn{5}{|l|}{ Pregnancy- related anxiety questionnaire (PRAQ) } \\
\hline English PRAQ Measure & 3681 & 21.23 & 5.26 & $8-40$ \\
\hline \multirow[t]{2}{*}{ French PRAQ-R2 Measure } & 789 & 25.71 & 7.84 & $10-48$ \\
\hline & $\mathrm{n}$ & $\%$ & & \\
\hline \multicolumn{5}{|l|}{ Disruptions to Prenatal Care } \\
\hline Changes in way prenatal care had been delivered & 4604 & $89.4 \%(n=4115)$ Yes & & \\
\hline Cancellation of prenatal care appointments & 4114 & $40 \%(n=1644)$ Yes & & \\
\hline $\begin{array}{l}\text { Support person allowed to attend prenatal } \\
\text { appointments }\end{array}$ & 4604 & $9.1 \%(n=421)$ Yes & & \\
\hline Changes to birth plan & 4604 & $33.3 \%(n=1535)$ Yes & & \\
\hline - $\quad$ Birth location & 4599 & $9.4 \%(n=434)$ Yes & & \\
\hline - $\quad$ Support person & 4599 & $26.7 \%(n=1229)$ Yes & & \\
\hline - Childcare plan & 4599 & $10.8 \%(n=499)$ Yes & & \\
\hline _ "other" changes & 4599 & $4.4 \%(n=204)$ Yes & & \\
\hline
\end{tabular}


Table 2. Prenatal Care Disruptions and Symptoms of Maternal Distress ${ }^{1}$

\begin{tabular}{|c|c|c|c|c|}
\hline & \multirow[t]{3}{*}{ Odds Ratio } & \multirow{2}{*}{\multicolumn{2}{|c|}{$\begin{array}{l}95 \% \text { CI for } \\
\text { Odds Ratio }\end{array}$}} & \multirow[t]{3}{*}{ p-value } \\
\hline & & & & \\
\hline & & Lower & Upper & \\
\hline \multicolumn{5}{|l|}{ General Anxiety Symptoms } \\
\hline Prenatal appointment cancelled & 1.33 & 1.17 & 1.52 & .000 \\
\hline Support person(s) not allowed to & .94 & .74 & 1.19 & .61 \\
\hline \multicolumn{5}{|l|}{ attend prenatal appointments } \\
\hline Birth location change & 1.11 & .88 & 1.39 & .38 \\
\hline Birth support person change & 1.77 & 1.49 & 2.09 & .000 \\
\hline Birth childcare changes & 1.48 & 1.16 & 1.90 & .002 \\
\hline \multicolumn{5}{|l|}{ Depression Symptoms } \\
\hline Prenatal appointment cancelled & 1.36 & 1.19 & 1.56 & .000 \\
\hline Support person(s) not allowed to & .87 & .67 & 1.12 & .280 \\
\hline \multicolumn{5}{|l|}{ attend prenatal appointments } \\
\hline Birth location change & 1.23 & .98 & 1.53 & .080 \\
\hline Birth support person change & 1.61 & 1.36 & 1.91 & .000 \\
\hline Birth childcare changes & 1.50 & 1.18 & 1.92 & .001 \\
\hline \multicolumn{5}{|l|}{ Pregnancy-Related Anxiety } \\
\hline \multicolumn{5}{|l|}{ Symptoms (English Measure) } \\
\hline Prenatal appointment cancelled & 1.39 & 1.17 & 1.64 & .000 \\
\hline
\end{tabular}




\begin{tabular}{|c|c|c|c|c|}
\hline $\begin{array}{l}\text { Support person(s) not allowed to } \\
\text { attend prenatal appointments }\end{array}$ & .815 & .60 & 1.11 & .190 \\
\hline Birth location change & 1.22 & .94 & 1.59 & .130 \\
\hline Birth support person change & 1.70 & 1.39 & 2.08 & .000 \\
\hline Birth childcare changes & 1.46 & 1.08 & 2.00 & .015 \\
\hline \multicolumn{5}{|l|}{ Pregnancy-Related Anxiety } \\
\hline \multicolumn{5}{|l|}{ Symptoms (French Measure) } \\
\hline Prenatal appointment cancelled & .91 & .63 & 1.32 & .628 \\
\hline $\begin{array}{l}\text { Support person(s) not allowed to } \\
\text { attend prenatal appointments }\end{array}$ & 1.50 & .73 & 2.92 & .284 \\
\hline Birth location change & .74 & .35 & 1.55 & .422 \\
\hline Birth support person change & 1.58 & 1.00 & 2.50 & .049 \\
\hline Birth childcare changes & .88 & .41 & 1.90 & .746 \\
\hline
\end{tabular}

$227{ }^{1}$ Model adjusted for maternal education, age, ethnicity, household income, parity and trimester 
References

230

231

232

233

234

235

236

237

238

239

240

241

242

243

244

245

246

247

248

249

250

251

252

253

254

255

256

257

258

259

260

261

262

263

264

265

266

267

268

269

270

271

272

273

274

275

1. Campbell, O.M.R., and Graham, W.J. Strategies for reducing maternal mortality: getting on with what works. Lancet (2006) 368:9543. doi: 10.1016/S0140-6736(06)69381-1.

2. Chen, X-K., Wen, S.W., Yang, Q., Walker, M.C. Adequacy of prenatal care and neonatal mortality in infants born to mothers with and without antenatal high-risk conditions. Aust. NZ. J. Obst. Gyn. (2007) 47:2 doi: 10.1111/j.1479-828X.2007.00697.x.

3. Hohmann-Marriott, B. The couple context of pregnancy and its effects on prenatal care and birth outcomes. Mat. Child. Health. J. (2009) 13:6. doi: 10.1007/s10995-009-0467-0.

4. Van Dijk, J.A.W., Anderko, L., Stetzer, F. The impact of prenatal care coordination on birth outcomes. J. Obst. Gyn. Neon. Nurs. (2011) 40:1. doi: 10.1111/j.1552-6909.2010.01206.x.

5. Partridge, S., Balayla, J., Holcroft, C.A., Abenhaim, H.A. Inadequate prenatal care utilization and risks of infant mortality and poor birth outcome: a retrospective analysis of 28,729,765 U.S. deliveries over 8 years. Am. J. Perinatol. (2012) 29:10. doi: 10.1055/s-0032-1316439.

6. Public Health Agency of Canada. (2020). Pregnancy, childbirth and caring for newborns: Advice for mothers during COVID-19. https://www.canada.ca/en/publichealth/services/publications/diseases-conditions/pregnancy-advise-mothers.html [Accessed December 15, 2020].

7. Lancaster, C.A., Gold, K.J., Flynn, H.A., Yoo, H., Marcus, S.M., Davis, M.M. Risk factors for depressive symptoms during pregnancy: a systematic review. Am. J. Obstet. Gyn. (2010) 202:1. doi: 10.1016/j.ajog.2009.09.007.

8. Leahy-Warren, P., McCarthy, G., Corcoran, P. First-time mothers: social support, maternal parental self-efficacy and postnatal depression. J. Clin. Nurs. (2012) 21:3-4. doi: 10.1111/j.1365-2702.2011.03701.x.

9. Dayan, J., Creveuil, C., Marks, M.N., Conroy, S., Herlicoviez, M., Dreyfus, M., et al. Prenatal depression, prenatal anxiety, and spontaneous preterm birth: a prospective cohort study among women with early and regular care. Psychosom. Med. 68:6 (2006). doi: 10.1097/01.psy.0000244025.20549.bd.

10. Waldenstrom, U. Experience of labor and birth in 1111 women. J. Psychosom. Res. (1999) 47:5, 471-82.

11. Bergant, A.M., Heim, K., Ulmer, H., Illmensee, K. Early postnatal depressive mood: associations with obstetric and psychosocial factors. J. Psychosom. Res. (1999) 46:4. doi: 10.1016/s0022-3999(98)00116-0.

12. Da Costa, D., Larouche, J., Dritsa, M., Brender, W. Psychosocial correlates of prepartum and postpartum depressed mood. J. Affect. Disord. (2000) 59:1. doi: 10.1016/s01650327(99)00128-7. 
13. Alipour, Z., Lamyian, M., Hajizadeh, E. Anxiety and fear of child-birth as predictors of postnatal depression in nulliparous women. Women and Birth. (2012) 25. doi: 10.1016/j.wombi.2011.09.002.

14. Ngai, F.W., and Ngu, S.F. Predictors of maternal and paternal depressive symptoms at postpartum. J. Psychosom. Res. (2015) 78. doi: 10.1016/j.jpsychores.2014.12.003.

15. Cornish, A.M., McMahon, C., Ungerer, J.A. Postnatal depression and the quality of motherchild interactions during the second year of life. Aust. J. Psychol. (2008) 60:3. doi: 10.1080/00049530701477738.

16. Rini, C.K., Dunkel-Schetter, C., Wadhwa, P.D., Sandman, C.A. Psychological adaptation and birth outcomes: the role of personal resources, stress, and sociocultural context in pregnancy. Health Psychol. (1999) 18:4. doi: 10.1037//0278-6133.18.4.333.

17. Huizink, A.C., Robles de Medina, P.G., Mulder, E.J.H., Visser, G.H.A., Buitelaar, J.K. Stress during pregnancy is associated with developmental outcome in infancy. J. Child Psychol. Psychiatry. (2003) 44:6. doi: 10.1111/1469-7610.00166.

18. Lobel, M., Cannella, D.L., Graham, J.E., DeVincent, C., Schneider, J., Meyer, B.A. Pregnancy-specific stress, prenatal health behaviors, and birth outcomes. Health Psychol. (2008) 27:5. doi: 10.1037/a0013242.

19. Dunkel-Schetter, C., and Lobel, M. Pregnancy and birth outcomes: a multilevel analysis of prenatal maternal stress and birth weight. Hand. Health Psych. (2012) 2:431-63.

20. Brockington I., Macdonald, E., Wainscott, G. Anxiety, obsessions and morbid preoccupations in pregnancy and the puerperium. Arch. Wom. Ment. Health. (2006) 9:5. doi: 10.1007/s00737-006-0134-z.

21. Phillips, J., Sharpe, L., Matthey, S., Charles, M. Maternally focused worry. Arch. Wom. Ment. Health. (2009) 12:6. doi: 10.1007/s00737-009-0091-4.

22. Pampaka, D., Papatheodorou, S.I., AlSeaidan, M., Al Wotayan, R., Wright, R.J., Buring, J.E., et al. Depressive symptoms and comorbid problems in pregnancy - results from a population based study. J. Psychosom. Res. (2018) 112. doi: 10.1016/j.jpsychores.2018.06.011

23. Lebel, C., MacKinnon, A., Bagshawe, M., Tomfohr-Madsen, L., Giesbrecht, G. Elevated depression and anxiety symptoms among pregnant individuals during the COVID-19 pandemic. J. Affect. Disord. (2020) 277. doi: 10.1016/j.jad.2020.07.126.

24. Davenport, M.H., Meyer, S., Meah, V.L., Strynadka, M.C., Khurana, R. Moms are not OK: COVID-19 and maternal mental health. Front. Glob. Womens Health. (2020) 1:1. doi: 10.3389/fgwh.2020.00001

25. Wu, Y., Zhang, C., Liu, H., Duan, C., Li, C., Fan, J., et al. Perinatal depressive and anxiety symptoms of pregnant women during the coronavirus disease 2019 outbreak in China. Am. J. Obstet. Gyn. (2020) 223:2. doi: 10.1016/j.ajog.2020.05.009. 
26. Ayaz, R., Hocaoglu, M., Gunay, T., Yardimci, O., Turgut, A., Karateke, A. Anxiety and depression symptoms in the same pregnant women before and during the COVID-19 pandemic. J. Perinatal Med. (2020) 48:9. doi: 10.1515/jpm-2020-0380.

27. Durankus, F., Aksu, E. Effects of the COVID-19 pandemic on anxiety and depressive symptoms in pregnant women: a preliminary study. J. Matern. Fetal Neonat. Med. (2020) 18. doi: $10.1080 / 14767058.2020 .1763946$.

28. Giesbrecht, G. Protocol for the Canadian Pregnancy During the COVID-19 Pandemic study [Preprint] (2020). Available at: https://psyarxiv.com/w8hd5/ (Accessed December 19, 2020).

29. Cox, J.L., Holden, J.M., Sagovsky, R. Detection of postnatal depression - development of the 10-item Edinburgh Postnatal Depression Scale. Brit. J. Psychiat. (1987) 150. doi: 10.1192/bjp.150.6.782

30. Kozinszky, Z., and Dudas, R.B. Validation studies of the Edinburgh Postnatal Depression Scale for the antenatal period. J. Affect. Disord. (2015) 176. doi: 10.1016/j.jad.2015.01.044.

31. Cella, D., Riley, W., Stone, A., Rothrock, N., Reeve, B., Yount, S., et al. The PatientReported Outcomes Measurement Information System (PROMIS) developed and tested its first wave of adult self-reported health outcome item banks: 2005-2008. J. Clin. Epidemiol. (2010) 63:11. doi: 10.1016/j.jclinepi.2010.04.011

32. Urizar, G.G., Yim, I.S., Rodriguez, A., Dunkel-Schetter, C. The SMART moms program: a randomized trial of the impact of stress management on perceived stress and cortisol in lowincome pregnant women. Psychoneuroendocrin. (2019) 104. doi: 10.1016/j.psyneuen.2019.02.022

33. Reymond, C., Derguy, C., Wendland, J., Loyal, D. French validation of a pregnancy-specific anxiety scale (PRAQ-R2). Psychological Practices. (2020) 26:3, 231-240.

34. Goletzke, J., Kocalevent, R-D., Hansen, G., Rose, M., Becher, H., Hecher, K., et al. Prenatal stress perception and coping strategies: insights from a longitudinal prospective pregnancy cohort. J. Psychosom. Res. (2017) 102. doi: 10.1016/j.jpsychores.2017.09.002.

35. Dayan, J., Creveuil, C., Herlicoviez, M., Herbel, C., Baranger, E., Savoye, C., et al. Role of anxiety and depression in the onset of spontaneous preterm labor. Am. J. Epidemiol. (2002) 155:4, 293-301.

36. Giammanco, M. D., and Gitto, L. Coping, uncertainty and health- related quality of life as determinants of anxiety and depression on a sample of hospitalized cardiac patients in Southern Italy. Qual. Life Res. (2016) 25. doi: 10.1007/s11136-016-1323-5.

37. Ng, J., Sham, A., Tang, P.L., Fung, S. SARS: pregnant women's fears and perceptions. Brit. J. Midwif. (2004) 12:11. doi: 10.12968/bjom.2004.12.11.16710

38. Walker, J. Control and the psychology of health. Philadelphia: Open University Press (2001). 
39. Simon, R.M., Johnson, K.M., Liddell, J. Amount, source, and quality of support as predictors of women's birth evaluations. Birth. (2016) 43:3. doi: 10.1111/birt.12227.

40. Madden, N., Emeruwa, U.N., Friedman, A.M., Aubey, J.J., Aziz, A., Baptiste, C.D., et al. Telehealth uptake into prenatal care and provider attitudes during the COVID-19 pandemic in New York City: a quantitative and qualitative analysis. Am. J. Perinatol. (2020) 37:10. doi: 10.11055/s-0040-1712939.

41. Hollander, J.E., Carr, B.G. Virtually perfect? Telemedicine for COVID-19. N. Engl. J. Med. (2020) 382:18. doi: 10.1056/NEJMp2003539. 